NTUA-03/02

hep-ph/0203095

\title{
Soft Supersymmetry Breaking from Coset Space Dimensional Reduction.
}

\author{
P. Manousselis ${ }^{a}$ and G. Zoupanos ${ }^{b}$ \\ Physics Department, National Technical University, \\ Zografou Campus, 15780 Athens, Greece.
}

\begin{abstract}
The Coset Space Dimensional Reduction scheme is briefly reviewed. Then a ten-dimensional supersymmetric $E_{8}$ gauge theory is reduced over symmetric and non-symmetric six-dimensional coset spaces. In general a four-dimensional non-supersymmetric gauge theory is obtained in case the used coset space is symmetric, while a softly broken supersymmetric gauge theory is obtained if the used coset space is non-symmetric. In the process of exhibiting the above properties we also present two attractive models, worth exploiting further, which lead to interesting GUTs with three families in four dimensions.
\end{abstract}

${ }^{a}$ e-mail address: pman@central.ntua.gr. Supported by ГГЕТ grand 97E $\Lambda / 71$.

${ }^{b}$ e-mail address: George.Zoupanos@cern.ch. Partially supported by EU under the RTN contract HPRN-CT-2000-00148 the A.v.Humboldt Foundation and the Greek-German Bilateral Programme IKYDA-2001. 


\section{Introduction}

In the recent years the theoretical efforts to establish a deeper understanding of Nature have managed to achieve a number of successes in developing frameworks that aim to describe the fundamental theory at the Planck scale. On the other hand a real breakthrough in a deeper knowledge of Nature should include an understanding of the, at present, free parameters of the Standard Model (SM) in terms of a few fundamental ones. Clearly the apparent success of the SM is spoiled by the presence of a plethora of free parameters mostly related to the adhoc introduction of the Higgs and Yukawa sectors in the theory. It is worth recalling that the Coset Space Dimensional Reduction (CSDR) [1, 2, 3, 4] was suggesting from the beginning that a unification of the gauge and Higgs sectors can be achieved in higher dimensions. The four-dimensional gauge and Higgs fields are simply the surviving components of the gauge fields of a pure gauge theory defined in higher dimensions. In the next step of development of the CSDR scheme, fermions were introduced [5, 6] and then the four-dimensional Yukawa and gauge interactions of fermions found also a unified description in the gauge interactions of the higher-dimensional theory. The last step in this unified description in high dimensions is to relate the gauge and fermion fields that have been introduced. A simple way to achieve that is to demand that the higher-dimensional gauge theory is $\mathcal{N}=1$ supersymmetric which requires that the gauge and fermion fields are members of the same supermultiplet.

In the spirit described above a very welcome additional input is that string theory suggests furthermore the dimension and the gauge group of the higher-dimensional supersymmetric theory [7]. Further support to this unified description comes from the fact that the reduction of the theory over coset [4] and CY spaces [7] provides the four-dimensional theory with scalars belonging in the fundamental representation of the gauge group as are introduced in the SM. In addition the fact that the SM is a chiral theory leads us to consider $D$-dimensional supersymmetric gauge theories with $D=4 n+2$ [8, 4], which include the ten dimensions suggested by the heterotic string theory [7].

Concerning supersymmetry, the nature of the four-dimensional theory depends on the corresponding nature of the compact space used to reduce the higher-dimensional theory. Specifically the reduction over CY spaces leads to supersymmetric theories 7 in four dimensions, the reduction over symmetric coset spaces leads to non-supersymmetric theories, while a reduction over non-symmetric ones leads to softly broken supersymmetric theories [9, 10, 11]. Concerning the latter as candidate four-dimensional theories that describe the nature, in addition to the usual arguments related to the hierarchy problem, we should remind a further evidence established in their favor the last years. It was found that the search for renormalization group invariant (RGI) relations among parameters of softly broken supersymmetric GUTs considered as a unification scheme at the quantum level, could lead to successful predictions in low energies. More specifically the search for RGI relations was concerning the parameters of softly broken GUTs beyond the unification point and could lead even to all-loop finiteness [12, 13]. On the other hand in the low energies lead to successful predictions not only for the gauge couplings but also for the top quark mass, among others, and to interesting testable predictions for the Higgs mass 14.

The review is organized as follows. In section 2 we describe the CSDR. In section 3 we describe two representative examples of CSDR of a ten-dimensional supersymmetric $E_{8}$ gauge theory over symmetric coset spaces. In section 1 we present the CSDR of the same $E_{8}$ gauge theory over the three existing non-symmetric coset spaces. Finally section 5 contains 
our conclusions.

\section{The Coset Space Dimensional Reduction (CSDR) scheme}

Let us proceed by recalling the fundamental ideas of the dimensional reduction procedure. Dimensional reduction is the construction of a lower dimensional Lagrangian starting from higher dimensions. In our construction we will use the symmetries of the extra dimensions. The CSDR is a dimensional reduction scheme where the extra dimensions form a coset space $S / R$ and the symmetry used is the group $S$ of isometries of $S / R$.

Given this form of the extra coordinates one can construct a lower dimensional Lagrangian by demanding the fields to be symmetric. This means that one requires the fields to be form invariant under the action of the group $S$ on extra coordinates,

$$
\delta_{\xi} \Phi^{i}=L_{\xi} \Phi^{i}=0
$$

where $L_{\xi}$ is the Lie derivative with respect to the Killing vectors $\xi$ of the extra dimensional metric. However this condition is too strong when the higher-dimensional Lagrangian possesses a symmetry. Then a generalized form of the symmetry condition (1) can be

$$
\delta_{\xi} \Phi^{i}=L_{\xi} \Phi^{i}=U_{\xi} \Phi^{i}
$$

where $U_{\xi}$ is the symmetry of the Lagrangian.

When we apply CSDR in a gauge theory the $U_{\xi}$ is a gauge transformation. Then the original Lagrangian becomes independent of the extra coordinates because of gauge invariance.

To be specific we consider a Yang-Mills-Dirac Lagrangian with gauge group $G$ in $D$ dimensions

$$
\begin{aligned}
S= & \int d^{D} x \sqrt{-g}\left[-\frac{1}{4} \operatorname{Tr}\left(F_{M N} F_{K \Lambda}\right) g^{M K} g^{N \Lambda}\right. \\
& \left.+\frac{i}{2} \bar{\psi} \Gamma^{M} D_{M} \psi\right],
\end{aligned}
$$

where capital indices $M, N$ run from $0 \ldots D-1$. The field strength is

$$
F_{M N}=\partial_{M} A_{N}-\partial_{N} A_{M}-\left[A_{M}, A_{N}\right]
$$

with $A_{M}$ the gauge field. $\Gamma^{M}$ are the $D$-dimensional gamma matrices satisfying

$$
\left\{\Gamma_{M}, \Gamma_{N}\right\}=2 g_{M N},
$$

$\psi$ is an anticommuting spinor in $D$ dimensions and $\bar{\psi}$ is the Dirac adjoint. $D_{M}$ is the covariant derivative,

$$
D_{M}=\partial_{M}-\theta_{M}-A_{M}
$$

with

$$
\theta_{M}=\frac{1}{2} \theta_{M N \Lambda} \Sigma^{N \Lambda}
$$


the spin connection of $M^{D}$.

Then the original spacetime $\left(M^{D}, g^{M N}\right)$, is assumed to be compactified to $M^{4} \times S / R$, with $S / R$ a coset space. The original coordinates $z^{M}$ become coordinates of $M^{4} \times S / R$, $z^{M}=\left(x^{m}, y^{\alpha}\right)$, where $\alpha$ is a curved index of the coset, and with $a$ we will denote a flat tangent space index. The metric is

$$
g^{M N}=\left[\begin{array}{cc}
\eta^{\mu \nu} & 0 \\
0 & -g^{\alpha \beta}
\end{array}\right],
$$

where $\eta^{\mu \nu}=\operatorname{diag}(1,-1,-1,-1)$ is the Minkowski spacetime metric and $g^{\alpha \beta}$ is the coset space metric.

To develop the geometry of coset spaces we divide the generators of $S, Q_{A}$ in two sets, the generators of $R, Q_{i}(i=1, \ldots, \operatorname{dim} R)$ and the generators of $S / R, Q_{a}(a=\operatorname{dim} R+$ $1 \ldots, \operatorname{dim} S)$. Then the commutation relations for the $S$ generators become

$$
\begin{aligned}
{\left[Q_{i}, Q_{j}\right] } & =f_{i j}^{k} Q_{k}, \\
{\left[Q_{i}, Q_{a}\right] } & =f_{i a}^{b} Q_{b}, \\
{\left[Q_{a}, Q_{b}\right] } & =f_{a b}^{i} Q_{i}+f_{a b}^{c} Q_{c} .
\end{aligned}
$$

The coset space $S / R$ is a symmetric one when $f_{a b}^{c}=0$.

The coordinates $y$ define an element of $S, L(y)$, which is a coset representative. Then the Maurer-Cartan form with values in the Lie algebra of $S$ is defined by

$$
V(y)=L^{-1}(y) d L(y)=e_{\alpha}^{A} Q_{A} d y^{\alpha}
$$

and obeys the Maurer-Cartan equation,

$$
d V+V \wedge V=0 .
$$

From the relation (11), and using standard techniques of differential geometry we can develop the geometry of coset spaces, and compute vielbeins, connections, curvature and torsion. For instance the vielbein and the $R$-connection can be computed at the origin $y=0$, and the results are, $e_{\alpha}^{a}=\delta_{\alpha}^{a}$ and $e_{\alpha}^{i}=0$.

Next we require that the fields are invariant up to a gauge transformation when $S$ acts on $S / R$. Let $\xi_{A}^{\alpha}, A=1, \ldots, \operatorname{dim} S$, be the Killing vectors which generate the isometries $S$ of $S / R$ and denote by $W_{A}$ the compensating gauge transformation associated with $\xi_{A}$. Define, next, the infinitesimal coordinate transformation

$$
\delta_{A} \equiv L_{\xi_{A}} .
$$

Then the condition that a coordinate transformation is compensated by a gauge transformation takes the following specific form when it is applied to the higher-dimensional vector and spinor

$$
\begin{aligned}
\delta_{A} A_{M} & =\xi_{A}^{\beta} \partial_{\beta} A_{M}+\partial_{M} \xi_{A}^{\beta} A_{\beta} \\
& =\partial_{M} W_{A}-\left[W_{A}, A_{M}\right], \\
\delta_{A} \psi & =\xi_{A}^{\alpha} \partial_{\alpha} \psi-\frac{1}{2} G_{A b c} \Sigma^{b c} \psi \\
& =D\left(W_{A}\right) \psi .
\end{aligned}
$$


Inspecting eq. (13) we recognize the familiar form of the Lie derivative of a vector and the gauge transformation appropriate for the adjoint representation. The same is true for the spinor in eq. (14). There $D\left(W_{A}\right)$ represents a gauge transformation in the representation to which the spinor belongs. The quantities $W_{A}$ may depend on internal coordinates $y$. The conditions (13), (14) should be covariant when $A_{M}(x, y)$ and $\psi(x, y)$ transform under a gauge transformation, therefore $W$ 's transform under a gauge transformation as

$$
\widetilde{W}_{A}=g W_{A} g^{-1}+\left(\delta_{A} g\right) g^{-1}
$$

Eqs (13), (14) and (15) provide us with the gauge freedom to do all calculations at $y=0$ and $W_{a}=0$. Note also that the variations $\delta_{A}$ satisfy $\left[\delta_{A}, \delta_{B}\right]=f_{A B}^{C} \delta_{C}$ leading to a further consistency condition for the $W$ 's

$$
\xi_{A}^{\alpha} \partial_{\alpha} W_{B}-\xi_{B}^{\alpha} \partial_{\alpha} W_{A}-\left[W_{A}, W_{B}\right]=f_{A B}{ }_{C} W_{C} .
$$

The above conditions imply certain constraints that the $D$-dimensional fields should obey. The solution of the constraints provide

- The remaining gauge invariance in four dimensions,

- The four-dimensional spectrum,

- The scalar potential of the four-dimensional theory.

More specifically to find the four-dimensional gauge group we embed $R$ in $G$

$$
\begin{aligned}
G & \supset R_{G} \times H, \\
H & =C_{G}\left(R_{G}\right) .
\end{aligned}
$$

Then $H$, the centralizer of the image of $R$ in $G$, is the four-dimensional gauge group.

The scalar fields that are obtained from the higher components of the vector field are obtained as follows. First we embed $R$ in $S$ and decompose the adjoint of $S$ under $R$

$$
\begin{aligned}
S & \supset R \\
\operatorname{adj} S & =\operatorname{adj} R+\sum s_{i} .
\end{aligned}
$$

Then we embed $R$ in $G$ and decompose the adjoint of $G$ under $R \times H$,

$$
\begin{aligned}
G \supset & R_{G} \times H \\
\operatorname{adj} G= & (\operatorname{adj} R, 1)+(1, \operatorname{adj} H) \\
& +\sum\left(r_{i}, h_{i}\right) .
\end{aligned}
$$

The rule is that when

$$
s_{i}=r_{i}
$$

i.e. when we have two identical representation of $R$ in the decompositions (18), (19) there is an $h_{i}$ multiplet of scalar fields that survives in four dimensions.

To find the representation of $H$ under which the four-dimensional fermions transform, we have to decompose the representation $F$ of $G$ to which the fermions belong, under $R_{G} \times H$, i.e.

$$
F=\sum\left(t_{i}, h_{i}\right)
$$


and the spinor of $S O(d)$ under $R$

$$
\sigma_{d}=\sum \sigma_{j}
$$

Then for each pair $t_{i}$ and $\sigma_{i}$, where $t_{i}$ and $\sigma_{i}$ are identical irreducible representations, there is an $h_{i}$ multiplet of spinor fields in the four-dimensional theory.

In order to obtain chiral fermions in four-dimensions we have to impose the Weyl condition in $D$ dimensions. In $D=4 n+2$ dimensions, which is the case of interest, the decomposition of the left handed, say spinor under $S U(2) \times S U(2) \times S O(d)$ is

$$
\sigma_{D}=\left(2,1, \sigma_{d}\right)+\left(1,2, \bar{\sigma}_{d}\right)
$$

So we have in this case the decompositions

$$
\sigma_{d}=\sum \sigma_{k}, \bar{\sigma}_{d}=\sum \bar{\sigma}_{k}
$$

Let us start from a vector-like representation $F$ for the fermions. In this case each term $\left(t_{i}, h_{i}\right)$ in eq.(20) will be either self-conjugate or it will have a partner $\left(\bar{t}_{i}, \bar{h}_{i}\right)$. According to the rule described in eqs. (20), (21) and considering $\sigma_{d}$ we will have in four dimensions left-handed fermions transforming as $f_{L}=\sum h_{k}^{L}$. Since $\sigma_{d}$ is non self-conjugate, $f_{L}$ is non self-conjugate too. Similarly from $\bar{\sigma}_{d}$ we will obtain the right handed representation $f_{R}=\sum \bar{h}_{k}^{R}$ but as $F$ is vector-like, $\bar{h}_{k}^{R} \sim h_{k}^{L}$. Therefore there will appear two sets of Weyl fermions with the same quantum numbers under $H$. This is already a chiral theory, but still one can go further and try to impose the Majorana condition in order to eliminate the doubling of the fermionic spectrum. If we had started with $F$ complex, we should have again a chiral theory since in this case $\bar{h}_{k}^{R}$ is different from $h_{k}^{L}$ ( $\sigma_{d}$ non self-conjugate). Starting with $F$ vector-like along with the Majorana condition will be used in our discussions. The Majorana condition can be imposed in $D=2,3,4+8 n$ dimensions and is given by $\psi=C \bar{\psi}^{T}$, where $C$ is the $D$-dimensional charge conjugation matrix. Majorana and Weyl conditions are compatible in $D=4 n+2$ dimensions. Then in our case if we start with Weyl-Majorana spinors in $D=4 n+2$ dimensions we force $f_{R}$ to be the charge conjugate to $f_{L}$, thus arriving in a theory with fermions only in $f_{L}$. Furthermore if $F$ is to be real, then we have to have $D=2+8 n$, while for $F$ pseudoreal $D=6+8 n$.

The potential is obtained from the internal components of the field strength. In CSDR they are given by

$$
F_{a b}=f_{a b}^{C} \phi_{C}-\left[\phi_{a}, \phi_{b}\right]
$$

so the potential is

$$
\begin{aligned}
V(\phi)= & -\frac{1}{4} g^{a c} g^{b d} \operatorname{Tr}\left(f_{a b}^{C} \phi_{C}-\left[\phi_{a}, \phi_{b}\right]\right) \\
& \times\left(f_{c d}^{D} \phi_{D}-\left[\phi_{c}, \phi_{d}\right]\right) .
\end{aligned}
$$

The minimization of the potential is in general a difficult problem. If however $S$ has an isomorphic image $S_{G}$ in $G$ which contains $R_{G}$, the minimum is zero. Furthermore, the fourdimensional gauge group $H$ breaks further by these non-zero vacuum expectation values of the Higgs fields to the centralizer $K$ of the image of $S$ in $G$, i.e. $K=C_{G}(S)$ [15]. More generally it can be proven [4] that dimensional reduction over a symmetric coset space always gives a potential of spontaneous breaking form. 
The effective action in four dimensions can be written as

$$
\begin{aligned}
S= & C \int d^{4} x\left(-\frac{1}{4} F_{\mu \nu}^{t} F^{t \mu \nu}\right. \\
& -\frac{1}{2}\left(D_{\mu} \phi_{\alpha}\right)^{t}\left(D^{\mu} \phi^{\alpha}\right)^{t}+V(\phi) \\
& \left.+\frac{i}{2} \bar{\psi} \Gamma^{\mu} D_{\mu} \psi-\frac{i}{2} \bar{\psi} \Gamma^{a} D_{a} \psi\right),
\end{aligned}
$$

where

$$
D_{\mu}=\partial_{\mu}-A_{\mu}
$$

is the spacetime part of the gauge covariant derivative and

$$
D_{a}=\partial_{a}-\theta_{a}-\phi_{a}
$$

is the internal part of the gauge covariant derivative, with

$$
\theta_{a}=\frac{1}{2} \theta_{a b c} \Sigma^{b c}
$$

the spin connection of the coset space. $C$ is the volume of the coset space.

Note that the second fermion term in eq. (26) can be written as

$$
L_{Y}=\frac{i}{2} \bar{\psi} \Gamma^{a} \nabla_{a} \psi+\bar{\psi} V \psi
$$

where

$$
\begin{aligned}
\nabla_{a} & =-\partial_{a}+\frac{1}{2} f_{i b c} e_{\gamma}^{i} e_{a}^{\gamma} \Sigma^{b c}+\phi_{a} \\
V & =\frac{i}{4} \Gamma^{a} G_{a b c} \Sigma^{b c} .
\end{aligned}
$$

In eq. (30) we have used the full connection with torsion,

$$
\begin{aligned}
\theta_{c b}^{a} & =-f_{i b}^{a} e_{\alpha}^{i} e_{c}^{\alpha}-\left(D_{c b}^{a}+\frac{1}{2} \Sigma_{c b}^{a}\right) \\
& =-f_{i b}^{a} e_{\alpha}^{i} e_{c}^{\alpha}-G_{c b}^{a}
\end{aligned}
$$

with

$$
D_{c b}^{a}=g^{a d} \frac{1}{2}\left[f_{d b}^{e} g_{e c}+f_{c b}^{e} g_{d e}-f_{c d}^{e} g_{b e}\right],
$$

and $\Sigma_{c b}^{a}$ the contorsion. The general choice of the contorsion tensor is

$$
\Sigma_{a b c}=2 \tau\left(D_{a b c}+D_{b c a}-D_{c b a}\right),
$$

where $\tau$ is a free parameter. Furthermore the constraints imply, that $\nabla_{a}=\phi_{a}$, when $\nabla_{a}$ acts on a spinor field, and the term $\frac{i}{2} \bar{\psi} \Gamma^{a} \nabla_{a} \psi$ in eq. (29) is exactly the Yukawa term. The last term, $V=\frac{i}{4} \Gamma^{a} G_{a b c} \Sigma^{b c}$ gives mass to the gaugino as will be explained in the next section. 


\section{Coset Space Dimensional Reduction over symmetric coset spaces}

Recently we have examined the CSDR of supersymmetric gauge theories in ten dimensions over all six-dimensional coset spaces [11]. Our results are that when the coset space is symmetric, there is no remnant of the supersymmetric spectrum in the four-dimensional theory. Here we present two examples representing this case, one is instructive and the other is a promising candidate to become a realistic model.

\subsection{CSDR over $S O(7) / S O(6)$}

The ten-dimensional gauge group that we consider is $G=E_{8}$. First we examine the dimensional reduction over $S / R=S O(7) / S O(6)$, which is a symmetric coset space. To apply the rules we need the embedding of $R=S O(6)$ in $G=E_{8}$ and the decomposition of the adjoint of $G$,

$$
\begin{aligned}
E_{8} \supset & S O(6) \times S O(10) \\
248= & (15,1)+(1,45)+(6,10) \\
& +(4,16)+(\overline{4}, \overline{16}) .
\end{aligned}
$$

Then the four-dimensional gauge group is $H=C_{E_{8}}(S O(6))=S O(10)$.

To find the $R=S O(6)$ content of $S O(7) / S O(6)$ vector and spinor we need the decompositions

$$
\begin{aligned}
S O(7) & \supset S O(6) \\
21 & =15+6
\end{aligned}
$$

and

$$
\begin{aligned}
S O(6) & \supset S O(6) \\
4 & =4 .
\end{aligned}
$$

Therefore the $R=S O(6)$ content of the vector and spinor of $S O(7) / S O(6)$ are 6 and 4 respectively.

The rules stated in section 2 are telling us that from the ten-dimensional gauge field $A_{M}^{a}$ we obtain in four dimensions the gauge field $A_{\mu}^{\alpha \beta}$ and the scalars $\chi^{m}$, where $a$ is a 248 $E_{8}$-index, $\alpha \beta$ a 45 and $m$ a $10 S O(10)$-index. From the ten-dimensional gaugino $\psi^{a}$, we obtain the four-dimensional left-handed matter fermions $\psi^{s}$, belonging to 16 of $S O(10)$. In summary

$$
\begin{aligned}
A_{M}^{a} & \longrightarrow A_{\mu}^{\alpha \beta}, \chi^{m}, \\
\psi^{a} & \longrightarrow \psi^{s} .
\end{aligned}
$$

Note that there is no supersymmetric partner of the four-dimensional gauge field and the scalar matter in four dimensions transforms as a 10-plet, while fermion matter as a lefthanded 16-plet, therefore the spectrum of the four-dimensional theory is non-supersymmetric. 


\subsection{CSDR over $C P^{2} \times S^{2}$}

Choosing $G=E_{8}$ and $S / R=C P^{2} \times S^{2}$ which is the coset $S U(3) \times S U(2) / S U(2) \times U(1) \times U(1)$ we have an interesting and promising example. The embedding of $R=S U(2) \times U(1) \times U(1)$ in $E_{8}$ is given by the decomposition

$$
\begin{aligned}
E_{8} \supset & S U(2) \times U(1) \times U(1) \times S O(10) \\
248= & (1,45)_{(0,0)}+(3,1)_{(0,0)}+(1,1)_{(0,0)} \\
& +(1,1)_{(0,0)}+(1,1)_{(2,0)}+(1,1)_{(-2,0)} \\
& +(2,1)_{(1,2)}+(2,1)_{(-1,2)} \\
& +(2,1)_{(-1,-2)}+(2,1)_{(1,-2)} \\
& +(1,10)_{(0,2)}+(1,10)_{(0,-2)} \\
& +(2,10)_{(1,0)}+(2,10)_{(-1,0)} \\
& +(2,16)_{(0,1)}+(1,16)_{(1,-1)} \\
& +(1,16)_{(-1,-1)}+(2, \overline{16})_{(0,-1)} \\
& +(1, \overline{16})_{(-1,1)}+(1, \overline{16})_{(1,1)} .
\end{aligned}
$$

The four-dimensional gauge group is $H=C_{E_{8}}(S U(2) \times U(1) \times U(1))=S O(10) \times U(1) \times U(1)$. The vector and spinor content under $R$ of the specific coset are, $1_{0,2 a}+1_{0,-2 a}+2_{b, 0}+2_{-b, 0}$ and $1_{b,-a}+1_{-b,-a}+2_{0, a}$ respectively. Choosing $a=b=1$ we find that the scalar fields of the four-dimensional theory transform as $10_{(0,2)}, 10_{(0,-2)}, 10_{(1,0)}, 10_{(-1,0)}$ under $H$. Also, we find that the fermions of the four-dimensional theory are the following left-handed multiplets of $H: 16_{(-1,-1)}, 16_{(1,-1)}, 16_{(0,1)}$. Therefore altogether we find

$$
\begin{aligned}
A_{M}^{a} & \longrightarrow A_{\mu}^{\alpha \beta}, \chi_{1}^{m}, \chi_{2}^{m}, \chi_{3}^{m}, \chi_{4}^{m} \\
\psi^{a} & \longrightarrow \psi_{1}^{s}, \psi_{2}^{s}, \psi_{3}^{s},
\end{aligned}
$$

where the index conventions are as in the previous subsection 3.1. Worth noting are the nonsupersymmetric spectrum as in the previous example as well as the three fermion generations which is a clear improvement as compared to the previous case.

Analogous conclusions concerning supersymmetry can be drawn by examining the rest six-dimensional symmetric coset spaces [11].

\section{Soft SUSY breaking by CSDR over non-symmetric coset spaces}

In the present section we examine the reduction of a ten-dimensional $E_{8}$ gauge theory over the three non-symmetric coset spaces. We find that a softly broken supersymmetric fourdimensional gauge theory is obtained.

\subsection{CSDR over $G_{2} / S U(3)$}

As first example of CSDR over a non-symmetric coset space, we present the case of $G_{2} / S U(3)$ [9]. The rest two six-dimensional non-symmetric coset spaces i.e. $S p(4) /(S U(2) \times U(1))_{\text {non.max }}$ 
and $S U(3) / U(1) \times U(1)$, are examined in the following subsections 4.2 and 4.3 according to refs. [10, 11].

We start again with $G=E_{8}$ in ten dimensions, $S / R=G_{2} / S U(3)$, and $R=S U(3)$. Now we need the decomposition

$$
\begin{aligned}
E_{8} & \supset S U(3) \times E_{6} \\
248 & =(8,1)+(1,78)+(3,27)+(\overline{3}, \overline{27}),
\end{aligned}
$$

from which we find that the four-dimensional gauge group is $H=C_{S U(3)}\left(E_{8}\right)=E_{6}$.

Next, to find the $R=S U(3)$ content of $G_{2} / S U(3)$ vector and spinor, we examine the decompositions

$$
\begin{aligned}
G_{2} & \supset S U(3) \\
14 & =8+3+\overline{3}
\end{aligned}
$$

and

$$
\begin{aligned}
S O(6) & \supset S U(3) \\
4 & =1+3
\end{aligned}
$$

from which we conclude that the $R=S U(3)$ content of $G_{2} / S U(3)$ vector and spinor are $3+\overline{3}$ and $1+3$ respectively. Then the CSDR rules determine that from the ten-dimensional gauge field $A_{M}^{a}$ we obtain in four dimensions the gauge field $A_{\mu}^{\alpha}$ and the scalars $\beta^{i}$, where $a$ is a $248 E_{8}$-index, $\alpha$ a 78 and $i$ a $27-E_{6}$ index. From the ten-dimensional gaugino $\psi^{a}$ we obtain the four-dimensional gaugino $\lambda^{\alpha}$ and the left-handed matter fermions $\psi_{\beta}^{i}$. In summary we find

$$
\begin{array}{r}
A_{M}^{a} \longrightarrow A_{\mu}^{\alpha}, \beta^{i}, \\
\psi^{a} \longrightarrow \lambda^{\alpha}, \psi_{\beta}^{i} .
\end{array}
$$

Note that the surviving fields are organized as four-dimensional $\mathcal{N}=1$ vector and chiral multiplets $V^{\alpha}$ and $B^{i}$.

Moreover the scalar potential, using the metric of $G_{2} / S U(3)$,

$$
g_{a b}=R^{2} \delta_{a b}
$$

where $R$ is the radius of $G_{2} / S U(3)$, was found [9] to be

$$
\begin{aligned}
V(\beta)= & \frac{8}{R^{4}}-\frac{40}{3 R^{2}} \beta^{2}-\left[\frac{4}{R} d_{i j k} \beta^{i} \beta^{j} \beta^{k}+h . c\right] \\
& +\beta^{i} \beta^{j} d_{i j k} d^{k l m} \beta_{l} \beta_{m} \\
& +\frac{11}{4} \sum_{\alpha} \beta^{i}\left(G^{\alpha}\right)_{i}^{j} \beta_{j} \beta^{k}\left(G^{\alpha}\right)_{k}^{l} \beta_{l} .
\end{aligned}
$$

In turn it was found that the $F$-terms are obtained from the superpotential

$$
\mathcal{W}(B)=\frac{1}{3} d_{i j k} B^{i} B^{j} B^{k}
$$


where $d_{i j k}$ is the $E_{6}$-symmetric invariant tensor [16].

Similarly the D-terms were found to be

$$
D^{\alpha}=\sqrt{\frac{11}{2}} \beta^{i}\left(G^{\alpha}\right)_{i}^{j} \beta_{j},
$$

where $\left(G^{\alpha}\right)_{i}^{j}$ are representation matrices for the 27 of $E_{6}$. The rest terms of the scalar potential that are not obtained from $F$ - or $D$ - terms belong to the scalar Soft Supersymmetry Breaking (SSB) part of the Lagrangian, given by

$$
L_{S S B}=-\frac{40}{3 R^{2}} \beta^{2}-\left[\frac{4}{R} d_{i j k} \beta^{i} \beta^{j} \beta^{k}+h . c\right] .
$$

The SSB sector is completed by the gaugino Mass $M$, which is obtained from the $V$ operator of eqs.(29), (30), and was found to be in the present case

$$
M=(1+3 \tau) \frac{6}{\sqrt{3} R} .
$$

\subsection{CSDR over $S p(4) /(S U(2) \times U(1))_{\text {non.max }}$}

This time we have again $G=E_{8}$ in ten dimensions but $S / R=S p(4) /(S U(2) \times U(1))_{\text {non.max }}$. The decomposition to be used is

$$
\begin{aligned}
E_{8} \supset & S U(2) \times U(1) \times E_{6} \\
248= & \left(3_{0}, 1\right)+\left(1_{0}, 1\right)+\left(1_{0}, 78\right) \\
& +\left(2_{3}, 1\right)+\left(2_{-3}, 1\right) \\
& +\left(2_{1}, 27\right)+\left(2_{-1}, \overline{27}\right) \\
& +\left(1_{-2}, 27\right)+\left(1_{2}, \overline{27}\right) .
\end{aligned}
$$

Thus the four-dimensional gauge group is

$$
H=C_{E_{8}}(S U(2) \times U(1))=E_{6} \times U(1) .
$$

Now the $R=S U(2) \times U(1)$ content of $S p(4) /(S U(2) \times U(1))_{\text {non-max }}$. vector and spinor are

$$
2_{1}+2_{-1}+1_{2}+1_{-2}
$$

and

$$
2_{1}+1_{0}+1_{-2},
$$

respectively. From the CSDR rules we read that from the higher-dimensional gauge field we obtain the four-dimensional gauge fields, $A_{\mu}^{\alpha}, A_{\mu}$, with $\alpha$ a $78 E_{6}$-index, and two complex scalar fields $\beta^{i}, \gamma^{i}$, with $i$ a $27 E_{6}$-index. From the ten-dimensional gaugino we obtain the four-dimensional gaugino $\lambda^{\alpha}, \lambda$, and two spinors $\psi_{\beta}^{i}$, and $\psi_{\gamma}^{i}$ belonging to the 27 of $E_{6}$, i.e.

$$
\begin{aligned}
A_{M}^{a} & \longrightarrow A_{\mu}^{\alpha}, A_{\mu}, \beta^{i}, \gamma^{i}, \\
\psi^{a} & \longrightarrow \lambda^{\alpha}, \lambda, \psi_{\beta}^{i}, \psi_{\gamma}^{i} .
\end{aligned}
$$


Note that the surviving fields can be organized as two vector multiplets $V^{\alpha}, V$ and two chiral multiplets $B^{i}$, and $C^{i}$.

As in the previous case, the Lagrangian is $\mathcal{N}=1$ supersymmetric supplemented by a soft SSB part. The F-terms of the supersymmetric part are obtained from superpotential

$$
\mathcal{W}\left(B^{i}, C^{j}\right)=\sqrt{\frac{5}{7}} d_{i j k} B^{i} B^{j} C^{k}
$$

while the scalar SSB part is,

$$
\begin{array}{r}
\mathcal{L}_{\text {scalarSSB }}=-\frac{6}{R_{1}^{2}} \beta^{i} \beta_{i}-\frac{4}{R_{2}^{2}} \gamma^{i} \gamma_{i} \\
+\left[4 \sqrt{\frac{10}{7}} R_{2}\left(\frac{1}{R_{2}^{2}}+\frac{1}{2 R_{1}^{2}}\right) d_{i j k} \beta^{i} \beta^{j} \gamma^{k}+h . c\right] .
\end{array}
$$

$R_{1}$ and $R_{2}$ are the coset space scales coming from the metric,

$$
g_{a b}=\operatorname{diag}\left(R_{1}^{2}, R_{1}^{2}, R_{2}^{2}, R_{2}^{2}, R_{1}^{2}, R_{1}^{2}\right)
$$

Finally the gaugino Mass calculated from the $V$ operator is,

$$
M=(1+3 \tau) \frac{R_{2}^{2}+2 R_{1}^{2}}{8 R_{1}^{2} R_{2}} .
$$

\subsection{CSDR over $S U(3) / U(1) \times U(1)$}

The $G=E_{8}$, ten-dimensional theory is now reduced over the last non-symmetric coset space $S / R=S U(3) / U(1) \times U(1)$. From the decomposition

$$
\begin{aligned}
E_{8} \supset & U(1)_{1} \times U(1)_{2} \times E_{6} \\
248= & 1_{(0,0)}+1_{(0,0)}+1_{\left(3, \frac{1}{2}\right)} \\
& +1_{\left(-3, \frac{1}{2}\right)}+1_{(0,-1)}+1_{(0,1)} \\
& +1_{\left(-3,-\frac{1}{2}\right)}+1_{\left(-3,-\frac{1}{2}\right)} \\
& +78_{(0,0)}+27_{\left(3, \frac{1}{2}\right)} \\
& +27_{\left(-3, \frac{1}{2}\right)}+27_{(0,-1)} \\
& +\overline{27}_{\left(-3,-\frac{1}{2}\right)}+\overline{27}_{\left(3,-\frac{1}{2}\right)} \\
& +\overline{27}_{(0,1)},
\end{aligned}
$$

we conclude that the four-dimensional gauge group is,

$$
H=C_{E_{8}}\left(U(1)_{1} \times U(1)_{2}\right)=U(1)_{1} \times U(1)_{2} \times E_{6} .
$$

The $R=U(1) \times U(1)$ content of $S U(3) / U(1) \times U(1)$ vector and spinor are

$$
\begin{array}{r}
\left(3, \frac{1}{2}\right)+\left(-3, \frac{1}{2}\right)+(0,-1)+\left(-3,-\frac{1}{2}\right) \\
+\left(3,-\frac{1}{2}\right)+(0,1),
\end{array}
$$


and

$$
(0,0)+\left(3, \frac{1}{2}\right)+\left(-3, \frac{1}{2}\right)+(0,-1)
$$

respectively.

Applying the CSDR rules we find that from the ten-dimensional gauge fields we obtain the four-dimensional gauge fields $A_{\mu}^{\alpha}$, with $\alpha$ a $78 E_{6}$-index, $A_{(1) \mu}, A_{(2) \mu}$, the two $U(1)$ gauge fields, three scalars $\alpha^{i}, \beta^{i}, \gamma^{i}$, with $i$ a 27 index, and three $E_{6}$ singlets but charged under the $U(1) \mathrm{s}, \alpha, \beta$, and $\gamma$. From the ten-dimensional gaugino we obtain the supersymmetric partners of the above scalars, i.e.

$$
\begin{aligned}
A_{M}^{a} \longrightarrow & A_{\mu}^{\alpha}, A_{(1) \mu}, A_{(2) \mu} \\
& \alpha^{i}, \beta^{i}, \gamma^{i}, \alpha, \beta, \gamma \\
\psi^{a} \longrightarrow & \lambda^{\alpha}, \lambda_{(1)}, \lambda_{(1)}, \\
& \psi_{\alpha}^{i}, \psi_{\beta}^{i}, \psi_{\gamma}^{i}, \psi_{\alpha}, \psi_{\beta}, \psi_{\gamma}
\end{aligned}
$$

Therefore the surviving fields are organized as three vector multiplets, $V^{\alpha}, V_{(1)}, V_{(2)}$, and six chiral multiplets, $A^{i}, B^{i}, C^{i}, A, B, C$.

As in the previous two cases the Lagrangian is softly broken supersymmetric. The $F$ terms come from the superpotential

$$
\begin{aligned}
\mathcal{W}\left(A^{i}, B^{j}, C^{k}, A, B, C\right)= & \sqrt{40} d_{i j k} A^{i} B^{j} C^{k} \\
& +\sqrt{40} A B C .
\end{aligned}
$$

The scalar SSB part of the Lagrangian is,

$$
\begin{aligned}
& \mathcal{L}_{\text {scalarSSB }}=\left(\frac{4 R_{1}^{2}}{R_{2}^{2} R_{3}^{2}}-\frac{8}{R_{1}^{2}}\right) \alpha^{i} \alpha_{i} \\
& +\left(\frac{4 R_{1}^{2}}{R_{2}^{2} R_{3}^{2}}-\frac{8}{R_{1}^{2}}\right) \bar{\alpha} \alpha \\
& +\left(\frac{4 R_{2}^{2}}{R_{1}^{2} R_{3}^{2}}-\frac{8}{R_{2}^{2}}\right) \beta^{i} \beta_{i}+\left(\frac{4 R_{2}^{2}}{R_{1}^{2} R_{3}^{2}}-\frac{8}{R_{2}^{2}}\right) \bar{\beta} \beta \\
& +\left(\frac{4 R_{3}^{2}}{R_{1}^{2} R_{2}^{2}}-\frac{8}{R_{3}^{2}}\right) \gamma^{i} \gamma_{i}+\left(\frac{4 R_{3}^{2}}{R_{1}^{2} R_{2}^{2}}-\frac{8}{R_{3}^{2}}\right) \bar{\gamma} \gamma \\
& +\left[\sqrt{2} 80\left(\frac{R_{1}}{R_{2} R_{3}}+\frac{R_{2}}{R_{1} R_{3}}+\frac{R_{3}}{R_{2} R_{1}}\right)\right. \\
& \quad \times d_{i j k} \alpha^{i} \beta^{j} \gamma^{k} \\
& +\sqrt{2} 80\left(\frac{R_{1}}{R_{2} R_{3}}+\frac{R_{2}}{R_{1} R_{3}}+\frac{R_{3}}{R_{2} R_{1}}\right) \\
& \quad \times \alpha \beta \gamma+h . c] .
\end{aligned}
$$

The coset space radii, $R_{1}, R_{2}, R_{3}$ are entering the above formula (65) through the metric, which takes the following form for the present coset $S U(3) / U(1) \times U(1)$

$$
g_{a b}=\operatorname{diag}\left(R_{1}^{2}, R_{1}^{2}, R_{2}^{2}, R_{2}^{2}, R_{3}^{2}, R_{3}^{2}\right)
$$


The SSB sector is completed by the gaugino mass,

$$
M=(1+3 \tau) \frac{\left(R_{1}^{2}+R_{2}^{2}+R_{3}^{2}\right)}{8 \sqrt{R_{1}^{2} R_{2}^{2} R_{3}^{2}}} .
$$

Thus we have established that besides the supersymmetric spectrum, we obtain a softly broken supersymmetric Lagrangian by CSDR over a non-symmetric coset space.

\section{Conclusions}

The CSDR was originally introduced as a scheme which, making use of higher dimensions, incorporates in a unified manner the gauge and the ad-hoc Higgs sector of the spontaneously broken gauge theories in four dimensions [1, 2]. Next fermions were introduced in the scheme and the ad-hoc Yukawa interactions have also been included in the unified description [5, 8].

Of particular interest for the construction of realistic theories in the framework of CSDR are the following virtues that complemented the original suggestion: (i) The possibility to obtain chiral fermions in four dimensions resulting from vector-like representations of the higher-dimensional gauge theory [8, 价. This possibility can be realized due the presence of non-trivial background gauge configurations which are introduced by the CSDR constructions [17], (ii) The possibility to deform the metric of certain non-symmetric coset spaces and thereby obtain more than one scales [. 1 , [1], (iii) The possibility to use coset spaces, which are multiply connected. This can be achieved by exploiting the discrete symmetries of

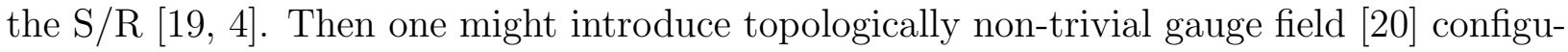
rations with vanishing field strength and induce additional breaking of the gauge symmetry. It is the Hosotani mechanism [21] applied in the CSDR.

In the above list recently has been added the interesting possibility that the popular softly broken supersymmetric four-dimensional chiral gauge theories might have their origin in a higher-dimensional supersymmetric theory with only vector supermultiplet [9, 10, 11], which is dimensionally reduced over non-symmetric coset spaces.

Let us also note that the current discussion on the higher-dimensional theories with large extra dimensions [22], provides a new framework to examine further the CSDR since the classical treatment used in CSDR is justified in the case of large radii which are far away from the scales that the quantum effects of gravity are important.

On the other hand we should also note that the effective field theories resulting from compactification of higher-dimensional theories contain also towers of massive higher harmonic (Kaluza-Klein) excitations, whose contributions at the quantum level alter the behaviour of the running couplings from logarithmic to power [23]. As a result the traditional pic-

ture of unification may change drastically [24, 25]. Combining the quantum behaviour of the higher-dimensional theories [24, 25], with the unification at the classical level of the gauge-Higgs and the gauge-Yukawa sectors that can be achieved in the CSDR scheme, one might hope to achieve a reduction of couplings of the SM by reducing the dimensions of a higher-dimensional theory.

\section{References}

[1] E. Witten, Phys. Rev. Lett. 38, 121(1977); N. S. Manton, Nucl. Phys. B 158, 141(1979). 
[2] P. Forgacs and N. S. Manton, Commun. Math. Phys. 72, 15(1980).

[3] Y. Kubyshin, J. M. Mourao, G. Rudolph and I. P. Volobujev, Lecture notes in Physics, Vol. 349, Springer Verlag, Heidelberg (1989).

[4] D. Kapetanakis and G. Zoupanos, Phys. Rept. C 219, 1(1992).

[5] N. S. Manton, Nucl. Phys. B 193, 502(1981).

[6] C. Wetterich, Nucl. Phys. B 222, 20 (1985); L. Palla, Z. Phys. C 24, 345(1983); K. Pilch and A. N. Schellekens, J. Math. Phys. 25, 3455(1984); P. Forgacs, Z. Horvath and L. Palla, Z. Phys. C 30, 261(1986); K. J. Barnes, P. Forgacs, M. Surridge and G. Zoupanos, Z. Phys. C 33, 427(1987).

[7] See e.g. M. B. Green, J. H. Schwarz and E. Witten, Superstring Theory, Cambridge University Press (1987); D. Luest and S. Theisen, Lectures on String Theory, Lecture Notes in Physics, Vol. 346, Springer Verlag, Heidelberg (1989).

[8] G. Chapline and R. Slansky, Nucl. Phys. B 204, 461(1982).

[9] P. Manousselis and G. Zoupanos, Phys. Lett. B 504, 122(2001).

[10] P. Manousselis and G. Zoupanos, Phys. Lett. B 518, 171(2001).

[11] P. Manousselis and G. Zoupanos, "Dimensional Reduction over Coset Spaces and Supersymmetry Breaking", JHEP 0203:002, (2002), hep-ph/0111125.

[12] D. Kapetanakis, M. Mondragón, and G. Zoupanos, Zeit. f. Phys. C 60, 181(1993); M. Mondragón, and G. Zoupanos, Nucl. Phys. B (Proc. Suppl.) 37 C, 98(1995).

[13] J. Kubo, M. Mondragón and G. Zoupanos, Nucl. Phys. B 424, 502(1994); ibid, Phys. Lett. B 389, 523(1996); T. Kobayashi et.al., Nucl. Phys. B 511, 45(1998); For an extended discussion and a complete list of references see: J. Kubo, M. Mondragón, and G. Zoupanos, Acta Phys. Polon. B 27 3991(1997).

[14] See e.g. T. Kobayashi et.al., Acta Phys. Polon. B 30 2013(1999); ibid., Proc. of HEP99, Tempere 1999, p.804.

[15] G. Chapline and N. S. Manton, Nucl. Phys. B 184, 391(1981); K. Farakos, G. Koutsoumbas, M. Surridge and G. Zoupanos, Nucl. Phys. B 291, 128(1987); ibid., Phys. Lett. B 191, 135(1987); Y. A. Kubyshin, J. M. Mourao, I. P. Volobujev, Int. J. Mod. Phys. A 4, 151(1989); F. A. Bais, K. J. Barnes, P. Forgacs and G. Zoupanos, Nucl. Phys. B 263, 557(1986).

[16] T. W. Kephart and M. T. Vaughn, Annals of Physics 145, 162(1983).

[17] S. Randjbar-Daemi, A. Salam and J. Strathdee, Nucl. Phys. B 242, 447(1983); Phys. Lett. B 124, 345(1983); P. Forgacs, Z. Horvath and L. Palla, Phys. Lett. B 147, 311(1984); B 148, 99(1984); A. N. Schellekens, Nucl. Phys. B 248, 706(1984); R. Coquereaux and A. Jadczyk, Commun. Math. Phys. 98, 79(1985). 
[18] B. E. Hanlon and G. C. Joshi, Phys. Lett. B 298, 312(1993).

[19] N. Kozimirov, V. A. Kuzmin and I. I. Tkachev, Sov. J. Nucl. Phys. 49, 164(1989); D. Kapetanakis and G. Zoupanos, Phys. Lett. B 232, 104(1989).

[20] G. Zoupanos, Phys. Lett. B 201, 301(1988).

[21] Y. Hosotani, Phys. Lett. B 126, 309(1983); B 129, 193(1983); E. Witten, Nucl. Phys. B 258, 75(1985); J. D. Breit, B. A. Ovrut and G. C. Segre, Phys. Lett. B 158, 33(1985); B. Greene, K. Kirlkin and P. J. Miron, Nucl. Phys. B 274, 575(1986); B. Greene, K. Kirlkin, P. J. Miron and G. G. Ross, Nucl. Phys. B 278, 667(1986).

[22] See e.g. I. Antoniadis and K. Benakli, "Large dimensions and string physics in future colliders", hep-ph/0007226 and references therein; Nima Arkani-Hamed, S. Dimopoulos and G. Dvali, Phys. Lett. B 249, 263(1998); Nima Arkani-Hamed, S. Dimopoulos and G. Dvali, Phys. Rev. D 59, 086004(1999); G. Dvali, S. Randjbar-Daemi and R. Tabbash, "The origin of spontaneous symmetry breaking in theories with large extra dimensions", hep-ph/0102307; Y. A. Kubyshin, "Models with Extra Dimensions and their Phenomenology", hep-ph/0111027.

[23] T. R. Taylor and G. Veneziano, Phys. Lett. B 212, 147(1988).

[24] K. R. Dienes, E. Dudas and T. Gherghetta, Nucl. Phys. B 537, 47(1999).

[25] T. Kobayashi, J. Kubo, M. Mondragon and G. Zoupanos, Nucl. Phys. B 550, 99(1999);

J. Kubo, H. Terao and G. Zoupanos, Nucl. Phys. B 574, 495(2000); ibid., hepph/0010069. 